\title{
Práticas educativas parentais: a percepção de adolescentes com e sem dependência química
}

\author{
Carla Zart Broecker - Pontifícia Universidade Católica do Rio Grande do Sul ${ }^{1}$ \\ Graciela Inchausti de Jou - Universidade Federal do Rio Grande do Sul
}

\begin{abstract}
Resumo
O uso de drogas vem acontecendo de forma cada vez mais precoce e com substâncias mais nocivas. Pesquisas nesta área procuram identificar os fatores de risco e de proteção, sendo que a família ou, mais especificamente, as práticas educativas parentais, podem atuar como ambos. O presente artigo teve como objetivo principal investigar a percepção das práticas educativas parentais de adolescentes com e sem diagnóstico de dependência química. Participaram da pesquisa 48 adolescentes de ambos os sexos, com idades variando entre 14 e 19 anos. Os participantes foram divididos em dois grupos (com e sem diagnóstico de dependência química) com 24 participantes cada um. Os dois grupos foram pareados de acordo com gênero, idade e nível socioeconômico. Foram aplicados dois instrumentos: o Questionário de Fatores de Risco para Dependência Química e o Parents' Report. Para a análise estatística foram utilizados o teste do Qui-Quadrado e o teste de Mann-Whitney. Os resultados mostraram que houve diferença significativa entre as percepções dos dois grupos: enquanto as práticas educativas parentais socialmente desejáveis estavam mais associadas aos participantes sem diagnóstico de dependência química, as práticas socialmente indesejáveis estavam mais associadas aos participantes com diagnóstico.

Palavras-chave: Práticas educativas parentais; Dependência química; Adolescência.
\end{abstract}

\section{Parental educative practices: the addicted and non addicted adolescents' perception}

\begin{abstract}
In the adolescence, the use of drugs has been happening at an earlier age and with harder drugs. Researches have tried to identify both risk and protection factors. Adolescents with or without diagnoses of chemical dependence had the perception of parental educative practices investigated in this study. There were 48 participants of both sexes, age variation from 14 to 19. They were divided in two groups: 1) 24 adolescents with diagnoses of dependence, 2) 24 adolescents without diagnosis. Both groups were paired according to gender, age and socialeconomic status. Two instruments were applied: The Questionnaire of Risk Factors for Chemical Dependence and The Parents' Report. In the statistical analysis were used Chi-Square and the Mann-Whitney tests. Results have shown significant difference between the perceptions of both groups, while practices more indicated socially were associated to adolescents without diagnoses, practices less indicated socially were associated to those with diagnoses. Keywords: Parental childrearing strategies; Drug dependence; Adolescence.
\end{abstract}

\section{Introdução}

O uso de drogas lícitas e ilícitas vem sendo foco de grande preocupação mundial e, embora seja um fenômeno antigo na história da humanidade, constitui um grave problema de saúde pública, com sérias conseqüências pessoais e sociais no futuro dos jovens e de toda a sociedade (Marques \& Cruz, 2000). O entendimento do uso de drogas sofre, portanto, interferências culturais, sociais, religiosas, políticas e científicas de cada população.

As primeiras experiências com drogas ocorrem, freqüentemente, na adolescência. Vários autores evidenciaram a precocidade quando identificam a faixa etária do início do uso de drogas dentro da adolescência, entre 10 e 19 anos, de acordo com a OMS (Baus, Kupek \& Pires, 2002; Carlini, Galduróz, Noto \& Nappo, 2001; Chaturvedi, Phukan \& Mahanta, 2003; De Micheli \& Formigoni, 2001; Huesca, Cruz, Encinas \& Pantoja, 2002; Kessler e cols., 2003; Pentz, 2003; Piko, 2000; Sanchez, Oliveira \& Nappo, 2004).

É na adolescência que as regras costumam ser questionadas e, até mesmo, contestadas, o que é necessário para o desenvolvimento da identidade dos jovens (Wagner, Carpenedo, Melo \& Silveira, 2005a). As influências do contexto social, somadas ao fato da adolescência ser uma época de experimentações, levam ao aparecimento de comportamentos de risco (Soldera, Dalgalarrondo, Filho \& Silva, 2004), entre eles, o uso de drogas lícitas e ilícitas.

\footnotetext{
${ }^{1}$ Endereço para correspondência:

Rua Tito Lívio Zambecari, 685/501 - 90450-231 - Porto Alegre-RS

E-mail: carlabroecker@terra.com.br
} 
Embora nem todas as pessoas que experimentam drogas se tornem dependentes, a dependência química é uma doença complexa, de tratamento longo e nem sempre eficaz. Sabe-se, ainda, que a dependência química é multideterminada (Marques \& Cruz, 2000), ou seja, devem ser considerados os inúmeros aspectos preditores (genéticos, psicológicos, familiares, sociais, entre outros), que, comumente, agem de modo associado.

Dentre os inúmeros fatores preditores da dependência química, a familia, ou, mais precisamente, as atitudes parentais para com os filhos, com o objetivo de educá-los, parece ser um aspecto passível de intervenção, principalmente ao pensar-se em prevenção. Dessa maneira, é importante olhar-se de modo mais acurado para o meio familiar, visando à compreensão dos fenômenos que ali ocorrem e buscando entender de que modo a familia pode ser protetora ou facilitadora dos comportamentos de risco dos filhos, inclusive do abuso de drogas e de uma possível dependência.

Pons (1998), por exemplo, detectou que um dos principais fatores protetores ao uso de drogas é a familia, pelo estabelecimento de laços afetivos entre seus membros, monitoramento das atividades e amizades do adolescente e construção de conduta social adequada. Nesta mesma linha de pesquisa, o estudo venezuelano de Rebolledo, Ortega e Pillon (2004) analisou 2.829 estudantes de ambos os sexos, com idades entre 12 e 17 anos, mediante o Test Drug Use Screening Inventory. Os autores constataram que a disfuncionalidade familiar e doença mental foram os fatores que estavam correlacionados com maior risco para o uso de drogas lícitas e ilícitas.

Pechansky, Szobot e Scivoletto (2004) também pesquisaram sobre a influência da família no uso de drogas dos jovens, constatando que, no que compete à situação familiar, a ausência do pai no domicilio do adolescente estava associada a um aumento de 22 vezes na chance deste ser dependente de drogas, quando comparado com adolescentes que viviam com ambos os pais. Corroborando estes achados, todos os tipos de traumas familiares, separação, brigas e agressões estavam francamente associados ao grupo de adolescentes com maior grau de dependência. Os mesmos autores acreditam, ainda, que o papel dos pais e do ambiente familiar é marcante no desenvolvimento do adolescente e, conseqüentemente, na sua relação com álcool e outras drogas. Falta de suporte parental, uso de drogas pelos próprios pais, atitudes permissivas dos pais perante o uso de drogas, incapacidade de controle dos filhos pelos pais, indisciplina e uso de drogas pelos irmãos são fatores que predispõem a iniciação ou o uso continuado de drogas por parte dos adolescentes.

A estrutura e o funcionamento familiar têm mudado muito nas últimas décadas. O modelo de família organizado com base na hierarquia, regido pela severidade de punição, foi substituído por formas diferenciadas de organização (Gomes \& Resende, 2004). Importantes fenômenos e movimentos sociais como a entrada das mulheres para o mercado de trabalho e sua maior participação no sistema financeiro familiar acabaram por imprimir um novo perfil à família. Wagner, Predebon, Mossmann e Verza (2005b) acreditam que, em contraponto à estrutura familiar tradicional, com o pai como único provedor e a mãe como única responsável pelas tarefas domésticas e cuidados com os filhos, o que vem ocorrendo na maioria das familias brasileiras de nível socioeconômico médio é um processo de transição. Atualmente, em muitas familias já se percebe uma relativa divisão de tarefas, na qual pais e mães compartilham aspectos referentes à educação dos filhos e à organização da rotina da família.

Dentre as tarefas que compõem a função parental, provavelmente a educação dos filhos seja a mais complexa (Wagner \& Bem, 2006). As modificações sociais e a repercussão destas na organização familiar tomaram a criação e educação dos filhos um grande e assustador desafio. Sabe-se que os modelos educativos que funcionaram para as gerações passadas já não podem ser aplicados atualmente, o que tem deixado os pais bastante confusos a respeito do que funciona, ou não, como técnica educativa. O que se percebe, portanto, são pais apreensivos e inseguros que se baseiam em tentativas e erros visando oferecer uma educação adequada para seus filhos.

No que se refere às práticas educativas, encontramse divergências entre alguns autores quanto a sua conceituação, havendo referência a termos como estilos, estratégias e práticas educativas. São chamadas práticas educativas as estratégias e técnicas utilizadas pelos pais e mães para orientar o comportamento de seus filhos, tentando atingir objetivos específicos em determinadas situações (Alvarenga \& Piccinini, 2001; Darling \& Steinberg, 1993; Wagner \& Bem, 2006). Para o presente estudo, foram adotados os termos práticas educativas socialmente desejáveis e práticas educativas socialmente indesejáveis, em função do instrumento utilizado que faz uso de tais nomenclaturas (Dibble \& Conhen, 1974, de acordo com Fischer \& Corcoran, 1994).

Em vista da necessidade social de uma maior orientação por parte dos profissionais, visando à eficiência dos pais em seu papel de educadores, é importante que se invista em pesquisas que respondam de modo mais objetivo sobre comportamentos parentais associados ao uso e nãouso de drogas pelos jovens (Carr \& Vandiver, 2001; Rebolledo e cols., 2004). Considerando que as atitudes dos pais em relação aos filhos podem possuir tanto a qualidade de proteção como a possibilidade de estímulo ao uso de drogas e à dependência das mesmas, este estudo buscou investigar a percepção dos adolescentes com e sem diagnóstico de dependência química a respeito das práticas

Psico-USF, v. 12, n. 2, p. 269-279, jul./ dez. 2007 
educativas de seus pais e analisar os aspectos sociobiodemográficos relacionados à dependência química na adolescência.

\section{Método}

\section{Participantes}

O presente estudo, de caráter exploratório e com abordagem quantitativa, teve como participantes 48 adolescentes, que foram divididos em dois grupos: grupo 1 , adolescentes com diagnóstico de dependência química ( $\mathrm{n}=$ 24), e grupo 2, adolescentes sem diagnóstico de dependência química $(\mathrm{n}=24)$. Os dois grupos foram pareados de acordo com gênero (mulheres: $33,3 \%$ e homens: 66,7\%), idade (idade média de 16,8 anos, com desvio padrão de 1,49 anos) e nível socioeconômico (baixo: 4,2\%, médio-baixo: $33,3 \%$ e médio: $62,5 \%$ ).

\section{Instrumentos}

Foram utilizados dois instrumentos para a investigação. O Parents' Report (PR), criado por Dibble e Conhen (1974, de acordo com Fischer \& Corcoran, 1994), traduzido e adaptado por Wagner, Grzybowski e Silveira (2003). O questionário avalia 8 categorias de práticas educativas socialmente desejáveis (aceitação da criança como pessoa, atenção centrada na criança, sensibilidade para sentimentos da criança, envolvimento positivo com a criança, aceitação da autonomia da criança, divisão da tomada de decisões com a criança, manutenção da disciplina de forma consistente e controle positivo da disciplina consideradas positivas) e 8 categorias de práticas educativas socialmente indesejáveis (desapego, intromissão, relaxamento do controle da disciplina, inconsistente manutenção da disciplina, controle através da ansiedade, controle através da culpa, controle através da autoridade, evitação da relação - consideradas negativas), havendo três itens de descrição comportamental para cada categoria.

Foi utilizado, também, o Questionário de Fatores de Risco para Dependência Química. O instrumento tem 27 questões e foi desenvolvido especialmente para este estudo pela pesquisadora. Um estudo piloto foi realizado para verificar a adequação das perguntas e a compreensão semântica das questões. Esse estudo contou com a participação de 5 meninas e 5 meninos com idades variando entre 12 e 19 anos. Esses adolescentes preencheram o questionário individualmente e assinalaram, no próprio material, dúvidas e sugestões que foram discutidas diretamente com as pesquisadoras. Foram descartadas as questões de difícil compreensão ou confusas, e foram incluídas algumas sugestões dos adolescentes para a facilitação do entendimento e do preenchimento do material. As questões elaboradas para o instrumento fundamentaram-se em uma revisão teórica que apontou as variáveis mais relacionadas ao uso de drogas por adolescentes.

\section{Procedimentos}

Para a coleta de dados, os participantes com diagnóstico de dependência química foram acessados através do centro para tratamento de dependência química de um hospital de Porto Alegre, onde os mesmos encontravam-se internados. Foi utilizado o critério de diagnóstico de dependência de substâncias do Manual diagnóstico e estatístico de transtornos mentais, DSM - IV (1995), que considera como dependente químico o sujeito que possui um agrupamento de sintomas cognitivos, comportamentais e fisiológicos indicando que o mesmo continua utilizando uma substância, apesar de problemas significativos relacionados a ela.

Após a pesquisa ter sido autorizada pela direção do local, os adolescentes que estavam internados no setor foram convidados a participar. Os adolescentes com 18 e 19 anos que aceitaram participar da pesquisa foram solicitados a assinar o Termo de Consentimento Livre e Esclarecido. Do mesmo modo, foram pedidas as autorizações dos pais dos adolescentes com idade inferior a 18 anos, para que eles pudessem fazer parte do estudo.

Após receber as autorizações, a pesquisadora marcou um horário individual para cada participante, em uma sala privativa dentro do próprio centro de tratamento. Com o objetivo de certificar-se de que os questionários seriam compreendidos e preenchidos adequadamente, a pesquisadora lia e preenchia, juntamente com o participante, os campos necessários. Primeiramente, foi preenchido o Questionário de Fatores de Risco e, posteriormente, o Parents' Report. Os participantes levaram, em média, 20 minutos para responder aos dois instrumentos.

A seleção dos participantes sem diagnóstico de dependência química foi feita por conveniência, através da rede social da pesquisadora. Após o contato com os adolescentes e a explicação da pesquisa, os adolescentes com 18 e 19 anos assinaram o Termo de Consentimento Livre e Esclarecido, tendo sido feito contato com os pais dos adolescentes com idade inferior a 18 anos, para que os mesmos assinassem o Termo de Consentimento autorizando a participação dos filhos na pesquisa. Posteriormente, contatou-se cada participante com a finalidade de marcar um horário conveniente no consultório da pesquisadora. A testagem ocorreu exatamente da mesma forma que foi descrita para os participantes com diagnóstico de dependência química.

\section{Procedimentos éticos}

O presente estudo passou por uma rigorosa aprovação do Comitê de Ética da PUCRS e tomou todos os cuidados necessários para a proteção dos participantes 
e pesquisadores. Foi utilizado o Termo de Consentimento Livre e Esclarecido, onde ficaram explicadas a proposta e as condições do estudo.

\section{Resultados}

Foi feita uma análise descritiva dos dados sociobiodemográficos dos participantes da pesquisa expostos na Tabela 1.

Tabela 1 - Dados sociobiodemográficos

\begin{tabular}{|c|c|c|}
\hline & Grupo DQ $(n=24)$ & Grupo SDQ ( $\mathrm{n}=24)$ \\
\hline Idade média & 16,8 & 16,8 \\
\hline Desvio padrão & 1,49 & 1,49 \\
\hline Sexo feminino & $33,3 \%$ & $33,3 \%$ \\
\hline Sexo masculino & $66,7 \%$ & $66,7 \%$ \\
\hline \multicolumn{3}{|l|}{ Classe socioeconômica } \\
\hline baixa & $42 \%$ & $4,2 \%$ \\
\hline média-baixa & $33,3 \%$ & $33,3 \%$ \\
\hline média & $62,5 \%$ & $62,5 \%$ \\
\hline \multicolumn{3}{|l|}{ Estuda } \\
\hline $\operatorname{sim}$ & $29,2 \%$ & $87,5 \%$ \\
\hline não & $70,8 \%$ & $12,5 \%$ \\
\hline \multicolumn{3}{|l|}{ Tipo de instituição em que estuda(va) } \\
\hline particular & $20,8 \%$ & $79,2 \%$ \\
\hline pública & $79,2 \%$ & $20,8 \%$ \\
\hline Repetiu de ano & $87,5 \%$ & $29,2 \%$ \\
\hline Trabalha & $37,5 \%$ & $33,3 \%$ \\
\hline Pratica alguma religião & $25 \%$ & $41,7 \%$ \\
\hline \multicolumn{3}{|l|}{ Com quem mora } \\
\hline pai, mãe e irmãos & $12,5 \%$ & $37,5 \%$ \\
\hline mãe, irmãos e outros & $25 \%$ & $25 \%$ \\
\hline pai, irmãos e outros & $12,5 \%$ & $4,2 \%$ \\
\hline outras configurações & $50 \%$ & $33,3 \%$ \\
\hline \multicolumn{3}{|l|}{ Estado civil dos pais } \\
\hline casados & $12,5 \%$ & $62,5 \%$ \\
\hline separados & $29,2 \%$ & $16,8 \%$ \\
\hline separados e recasados & $50 \%$ & $12,5 \%$ \\
\hline viúvos & $8,4 \%$ & $8,4 \%$ \\
\hline \multicolumn{3}{|l|}{ Número de irmãos } \\
\hline nenhum & ---------- & $25 \%$ \\
\hline um & $33,3 \%$ & $50 \%$ \\
\hline dois & $45,8 \%$ & $12,5 \%$ \\
\hline três & $12,5 \%$ & $12,5 \%$ \\
\hline quatro ou mais & $8,4 \%$ & -------- \\
\hline \multicolumn{3}{|l|}{ Posição na família } \\
\hline filho mais velho & $62,5 \%$ & $45,8 \%$ \\
\hline segundo mais velho & $12,5 \%$ & $12,5 \%$ \\
\hline terceiro mais velho & $4,2 \%$ & --------- \\
\hline filho mais novo & $20,8 \%$ & $16,7 \%$ \\
\hline filho único & --------- & $25 \%$ \\
\hline \multicolumn{3}{|l|}{ O que far no tempo livre } \\
\hline esporte & $25 \%$ & $42 \%$ \\
\hline fico com amigos & $54,2 \%$ & $54,2 \%$ \\
\hline saio para dançar - festas & $16,8 \%$ & $33,3 \%$ \\
\hline vejo televisão & $33,3 \%$ & $42 \%$ \\
\hline namoro & $33,3 \%$ & $54,2 \%$ \\
\hline computador - internet & $8,4 \%$ & $33,3 \%$ \\
\hline
\end{tabular}

Grupo DQ = com diagnóstico de dependência química

Grupo SDQ = sem diagnóstico de dependência química 
Foi feita uma análise descritiva acerca do padrão de uso de substâncias ilícitas dos partici-

Tabela 2 - Padrão de uso de substâncias ilícitas

\begin{tabular}{|c|c|c|}
\hline & Grupo DQ $(n=24)$ & Grupo SDQ $(\mathrm{n}=24)$ \\
\hline \multicolumn{3}{|l|}{ Tipos de drogas ilícitas consumidas* } \\
\hline maconha & $96 \%$ & $33,3 \%$ \\
\hline cocaína & $50 \%$ & $16,7 \%$ \\
\hline crack. & $79,2 \%$ & $8,4 \%$ \\
\hline loló & $41,7 \%$ & $8,4 \%$ \\
\hline lança-perfume & $4,2 \%$ & $12,5 \%$ \\
\hline ecstasy & --------- & $12,5 \%$ \\
\hline \multicolumn{3}{|l|}{ Quem oferecen drogas ilícitas } \\
\hline um desconhecido & $25 \%$ & $20,8 \%$ \\
\hline um conhecido ou amigo & $75 \%$ & $37,5 \%$ \\
\hline \multicolumn{3}{|l|}{ Onde ofereceram drogas ilicitas } \\
\hline na rua & $37,5 \%$ & $4,2 \%$ \\
\hline na vizinhança & $29,2 \%$ & $20,8 \%$ \\
\hline em casa & $12,5 \%$ & ---------- \\
\hline numa festa & $29,2 \%$ & $41,7 \%$ \\
\hline na escola & $4,2 \%$ & $16,7 \%$ \\
\hline \multicolumn{3}{|l|}{ Com quem usa drogas ilícitas } \\
\hline com amigos & $66,7 \%$ & $29,2 \%$ \\
\hline sozinho & $33,3 \%$ & $8,4 \%$ \\
\hline \multicolumn{3}{|l|}{ Quantos amigos seus usam drogas ilicitas } \\
\hline nenhum & --------- & $25 \%$ \\
\hline alguns & $12,5 \%$ & $58,3 \%$ \\
\hline metade & $20,8 \%$ & --------- \\
\hline muitos & $45,8 \%$ & $16,7 \%$ \\
\hline todos & $20,8 \%$ & ---------- \\
\hline Uso de drogas ilícitas na família & $37,5 \%$ & $16,7 \%$ \\
\hline \multicolumn{3}{|l|}{ Freqüência do uso de drogas ilícitas } \\
\hline uma vez por mês & $4,2 \%$ & $4,2 \%$ \\
\hline uma vez por semana & $8,4 \%$ & ---------- \\
\hline várias vezes por semana & $33,3 \%$ & 8,3 \\
\hline todos os dias & $54,2 \%$ & --------- \\
\hline \multicolumn{3}{|l|}{ O que pensa sobre seu consumo de drogas ilícitas } \\
\hline não tenho problema algum & ---------- & $87,5 \%$ \\
\hline deveria usar menos & --------- & $12,5 \%$ \\
\hline deveria parar de usar & $12,5 \%$ & ---------- \\
\hline sou dependente e preciso de ajuda & $87,5 \%$ & ---------- \\
\hline
\end{tabular}

Grupo DQ = com diagnóstico de dependência química

Grupo SDQ = sem diagnóstico de dependência química

${ }^{*} \mathrm{O}$ uso das drogas listadas pôde ser feito de forma conjunta, o que justifica a porcentagem superior a $100 \%$.

Os resultados mostraram que a maioria dos participantes $(70,8 \%)$ do grupo de dependentes químicos (DQ) não está estudando, 79,2\% destes freqüentam ou freqüentaram escolas públicas e $87,5 \%$ repetiram de ano pelo menos uma vez na vida.

Dos participantes do grupo sem dependência química (SDQ), 87,5\% estão estudando, 79,2\% freTabela 2. pantes. Estes dados encontram-se ilustrados da 
A respeito das atividades que os participantes realizam, os resultados mostraram que, do grupo de DQ, $37,5 \%$ trabalham, $25 \%$ praticam esportes, $54,2 \%$ costumam ficar com amigos, $16,8 \%$ vão a festas ou saem para dançar e $8,4 \%$ ficam navegando na internet ou utilizando o computador, $33,3 \%$ têm costume de olhar televisão nas horas livres e 33,3\% usam o tempo livre para namorar.

Quanto ao grupo de SDQ, 33,3\% trabalham, $42 \%$ praticam esportes, $54,2 \%$ costumam ficar com amigos, $33,3 \%$ vão a festas ou saem para dançar, $33,3 \%$ ficam navegando na internet ou utilizando o computador, $42 \%$ têm costume de olhar televisão nas horas livres e $54,2 \%$ usam o tempo livre para namorar.

Com relação à religiosidade, a maioria dos participantes do grupo de SDQ $(75 \%)$ não possui atividade religiosa, enquanto, no mesmo sentido, 58,3\% dos participantes do grupo de DQ também não praticam religião alguma.

Ao que se refere à experiência com drogas ilícitas dos participantes com e sem diagnóstico de dependência química, foram apontados os seguintes dados: no grupo de DQ 96\% usam maconha, 79,2\% usam crack, $50 \%$ usam cocaína, $41,7 \%$ usam loló (cola de sapateiro) e 4,2\% usam lança-perfume (éter). Já no grupo de SDQ, 33,3\% usam maconha, 16,7\% usam cocaína, $12,5 \%$ usam lança-perume e ecstasy e $8,4 \%$ usam crack e loló.

Entre os adolescentes do grupo de DQ, $75 \%$ apontaram que as drogas ilícitas foram oferecidas por amigos, $66,7 \%$ que foi na rua e $29,2 \%$ em festas. Já $37,5 \%$ dos adolescentes do grupo SDQ apontaram que as drogas ilícitas foram oferecidas por amigos, $25 \%$ que foi na rua e $41,7 \%$ em festas.

Entre os adolescentes do grupo de DQ, 66,7\% indicaram que as drogas são usadas com amigos e $54,2 \%$ faz uso de drogas ilícitas diariamente. Já entre os adolescentes do grupo de SDQ, 29,2\% indicaram que as drogas são usadas com amigos e $8,3 \%$ usa drogas ilícitas algumas vezes por semana.

Quanto a sua autopercepção com relação à drogadição, 87,5\% dos DQ apontaram ser dependentes químicos e admitiram precisar de ajuda, e $87,5 \%$ dos SDQ disseram não ter problemas em relação ao uso de drogas ilícitas.

Com a finalidade de verificar a associação entre as variáveis sociobiodemográficas e dependência química, foi utilizado o teste do Qui-Quadrado $\left(\mathrm{x}^{2}\right)$ e o nível de significância foi de $5 \%$. Os resultados mostraram que as variáveis não estudar $(\mathrm{p}=0,000$; $\left.\chi^{2}=16,800\right)$, repetir algum ano escolar $(\mathrm{p}=0,000$; $\left.\chi^{2}=16,800\right)$, estudar em escola pública $(\mathrm{p}=0,000$; $\left.\chi^{2}=24,929\right)$, ter pais separados $\left(\mathrm{p}=0,002 ; \chi^{2}=24,600\right)$, alguém ter oferecido drogas ilícitas $(\mathrm{p}=0,009$; $\left.\chi^{2}=7,111\right)$, drogas ilícitas terem sido oferecidas por um amigo ( $\left.\mathrm{p}=0,033 ; \chi^{2}=13,685\right)$, drogas ilícitas oferecidas na rua $\left(\mathrm{p}=0,045 ; \chi^{2}=18,674\right)$, muitos amigos fazerem uso de drogas ilícitas $\left(p=0,000 ; \chi^{2}=26,384\right)$, uso de drogas ilícitas na companhia de alguns amigos $(\mathrm{p}=0,001$; $\left.\chi^{2}=30,571\right)$ e uso combinado de maconha, cocaína e crack $\left(\mathrm{p}=0,000 ; \chi^{2}=41,333\right)$ têm associação estatisticamente significativa com a dependência química na adolescência.

Com a finalidade de verificar se há diferença entre os grupos com e sem diagnóstico de dependência química, em relação à percepção das práticas educativas parentais, foi utilizado o teste de Mann-Witney. Os resultados mostraram diferença estatisticamente significativa entre os dois grupos. $O$ nível de significância utilizado para a análise foi de $5 \%$.

Quatro das oito práticas socialmente indesejáveis (intromissão, controle através da culpa e da autoridade, evitacão da relação e manutenção inconsistente da disciplina) estão associadas à percepção dos adolescentes com dependência química a respeito das práticas de seus pais, enquanto quatro das oito práticas socialmente desejáveis (sensibilidade para os sentimentos dos filhos, envolvimento positivo, controle positivo da disciplina e divisão da tomada de decisões) estão associadas à percepção dos adolescentes sem diagnóstico. No entanto, cabe ressaltar que a prática socialmente indesejável manutenção inconsistente da disciplina está associada à percepção dos adolescentes sem diagnóstico. Esses dados encontram-se expostos na Tabela 3.

\section{Discussão}

Os resultados serão discutidos a partir da literatura que embasou este estudo, buscando analisar os dados obtidos nesta pesquisa. Em um primeiro momento, serão discutidas as práticas educativas parentais segundo a percepção dos adolescentes com diagnóstico clínico e sem diagnóstico clínico. Posteriormente, discute-se sobre fatores sociodemográficos relacionados aos grupos investigados.

Com relação às práticas educativas, sabe-se que estas são fruto da interação entre os pais e os filhos. Geralmente, os comportamentos educativos dos pais mudam de acordo com o comportamento do filho. Contudo manteve-se presente nesta análise que os comportamentos dos pais podem ser considerados tanto um fator de risco como de prevenção para a dependência química (Duvicq, Pereira \& Carvalho, 2004). 
Tabela 3 - Diferença na percepção das práticas educativas parentais dos participantes com e sem diagnóstico de dependência química

\begin{tabular}{|c|c|c|c|}
\hline \multirow{2}{*}{ Variáveis } & DQ & SDQ & $\mathrm{p}$ \\
\hline & \multicolumn{2}{|c|}{ Mean Rank } & \\
\hline Intromissão (PSI) & 29,94 & 19,06 & 0,005 \\
\hline Controle através da culpa (PSI) & 30,17 & 18,83 & 0,004 \\
\hline Controle através da autoridade (PSI) & 31,69 & 17,31 & 0,000 \\
\hline Evitação da relação (PSI) & 28,88 & 20,13 & 0,024 \\
\hline Manutenção inconsistente da disciplina (PSI) & 20,58 & 28,42 & 0,043 \\
\hline Sensibilidade para os sentimentos dos filhos (PSD) & 20,35 & 28,65 & 0,031 \\
\hline Envolvimento positivo (PSD) & 18,40 & 20,60 & 0,002 \\
\hline Aceitação da autonomia (PSD) & 17,81 & 31,19 & 0,001 \\
\hline Controle positivo da disciplina (PSD) & 18,00 & 31,00 & 0,001 \\
\hline Divisão da tomada de decisões (PSD) & 19,08 & 29,92 & 0,006 \\
\hline
\end{tabular}

$\mathrm{DQ}=$ grupo com diagnóstico de dependência química

$\mathrm{SDQ}=$ grupo sem diagnóstico de dependência química

PSD $=$ práticas socialmente desejáveis

PSI = práticas socialmente indesejáveis

Nível de significância $=5 \%$

As práticas socialmente indesejáveis apontadas pelos adolescentes com dependência química foram: intromissão, controle através da culpa, controle através da autoridade e evitação de relações. Já as práticas desapego, relaxamento no controle da disciplina, manutenção inconsistente da disciplina e controle através da ansiedade não foram mencionadas significativamente por esses adolescentes. A intromissão, o controle através da culpa e o controle através da autoridade estão relacionados a pais autoritários. De acordo com Costa, Teixeira e Gomes (2000), Darling e Steinberg (1993), Maccoby e Martin (1983), Patock-Peckham, Cheong, Balhorn e Nagoshi, (2001); Steinberg, Darling e Fletcher (1995) e Weber, Viezzer e Brandenburg (2004a), pais autoritários apresentam muitas regras e limites, mas são pouco afetivos e envolvem-se pouco com os filhos. São pais centrados em si próprios, portanto, desejam somente a obediência dos filhos. Geralmente, os pais com esses tipos de atitudes caracterizam-se por nível baixo de apoio e atenção emocional, são demasiadamente exigentes, tendo como resposta comum "porque eu disse assim", querem que o filho faça o que eles desejam, comandam a vida dos filhos e não deixam que ele próprio se expresse.

As práticas educativas parentais citadas anteriormente (intromissão, controle através da culpa e controle através da autoridade) tendem a gerar conseqüências na vida dos filhos. Os filhos podem apresentar desempenho moderado na escola e, se a coerção for muito forte, podem ter ansiedade, baixando o desempenho escolar e demonstrando hostilidade e agressividade contra figuras de autoridade (professores, por exemplo). Os filhos de pais com esses tipos de práticas educativas apresentam, ainda, piores desempenhos em habilidades sociais, humor instável, sendo pouco amigáveis, baixa auto-estima e altos níveis de depressão, situações que podem levá-los ao uso pesado de drogas como forma esquiva a esses problemas (Aunola, Stattin \& Nurmi, 2000; Baumrind, 1966; Miller, DiIorio \& Dudley, 2002).

A evitação da relação, outra prática apontada pelos adolescentes com dependência química, pode pressupor inexistência de relacionamento afetuoso entre pais e filhos ou pouco interesse, por parte dos pais, pelas atividades desenvolvidas pelos adolescentes (Duvicq e cols., 2004). Alguns autores denominam negligência tal conjunto de atitudes, considerando esses pais ausentes (Cohen, \& Rice, 1997; Piko, 2000; Steinberg, Lamborn, Darling, Mounts \& Dornbusch, 1994). Os autores colocam, ainda, que esses pais esperam que o filho responda as suas necessidades, formando famílias instáveis (com separações e conciliações freqüentes); são pais pouco presentes na vida dos filhos, não têm tolerância e aborrecem-se facilmente.

Os filhos criados neste contexto apresentam pior performance em todas as áreas, podendo ter um desenvolvimento atrasado e problemas afetivos e comportamentais. A evitação da relação, segundo Piko (2000) e De Micheli e Formigoni (2001), correlaciona-se com uso de drogas ilícitas e álcool, com doenças sexualmente transmissíveis, com início precoce da vida sexual, baixa auto-estima e auto-eficácia, com probabilidade maior de depressão, estresse, estilo explicativo pessimista, baixo desempenho acadêmico, 
baixas habilidades sociais e futuros comportamentos anti-sociais (mentir, roubar, agredir, etc.).

Contrastando com o quadro anterior, as práticas educativas sociamente desejáveis, como: envolvimento positivo, aceitação da autonomia, controle positivo da disciplina e divisão da tomada de decisões, ocorreram em maior freqüência no grupo SDQ, quando comparado com o DQ, de forma estatisticamente significativa associadas ao grupo sem diagnóstico de dependência química.

Em se tratando das práticas educativas socialmente desejáveis, a sensibilidade para os sentimentos dos filhos e o envolvimento positivo, que também podem ser traduzidos como expressão do afeto, apoio e compreensão, são entendidos como aspectos preventivos poderosos em relação ao abuso de substâncias psicoativas e aos problemas de conduta (Dekovic, Janssens \& Van As, 2003; Wu, Lu, Sterling \& Weisner, 2004). Segundo Pons (1998), os filhos de pais que se utilizam de tais práticas sentem-se aceitos, valorizados e seguros, percepções fundamentais no desenvolvimento das relações sociais dos adolescentes.

De acordo com alguns pesquisadores (Oliveira, Marin \& Pires, 2002; Salvador \& Weber, 2005; Sidman, 2001), o controle positivo da disciplina, prática desejável que também se mostrou significativa $(p=0,001)$ no grupo sem dependência química, pode ser utilizado pelos pais para incentivar e fortalecer comportamentos desejáveis em filhos, como também para desestimular e substituir os indesejáveis. Alvarenga (2001) cita que o uso de reforçadores positivos e regras fazem com que os filhos tenham uma melhor adaptação na vida social, aprendendo a respeitar e seguir regras, compreendendo a importância destas.

Quanto à aceitação da autonomia e à divisão da tomada de decisões, Gomide (2003) aponta que, no convívio diário, os pais devem procurar direcionar o comportamento dos filhos com o objetivo de seguir certos princípios morais e adquirir uma ampla gama de comportamentos que garantam independência, autonomia e responsabilidade, para que, posteriormente, possam desempenhar adequadamente seu papel social.

As respostas em relação à manutenção inconsistente da disciplina (prática socialmente indesejável) foram estatisticamente diferentes, quando comparados os dois grupos. O fato de ter se apresentado mais presente no grupo sem diagnóstico de dependência química pode ser interpretado pela presença mais efetiva dos pais, que apresentam muito afeto e envolvimento e poucas regras e limite. Esses pais costumam dar muito apoio e atenção emocional, mas pouca estrutura positiva e direção aos filhos. Geralmente os filhos têm boa auto-estima e boas habilidades sociais, com baixos níveis de depressão, embora possam colocar-se em situações de risco por não terem aprendido que existem regras e limites no mundo (Pentz, 2003; Weber, Brandenburg, \& Viezzer, 2003; Weber, Prado, Viezzer, \& Brandenburg, 2004a). Deve-se lembrar que a maioria dos adolescentes caracterizados sem dependência química também, de uma forma ou outra, podem fazer drogas lícitas ou ilícitas.

Em relação à associação dos dados sociobiodemográficos e dependência química, a separação dos pais aparece como fator significativo em relação à predisposição ao uso de drogas. Vários autores identificaram níveis de risco no uso de drogas em jovens pertencentes a familias com pais separados ou famílias com relacionamentos muito deteriorados (Piko, 2000, entre outros). Dessa forma, o presente estudo corrobora pesquisas anteriores que identificaram associação entre o uso de drogas e separação dos pais.

A associação entre as variáveis não estudar, repetência escolar e dependência química também foi encontrada em um estudo de Tavares, Beria e Lima (2001). Esses pesquisadores constataram que alunos que faltaram nove ou mais dias no mês anterior, ou que tiveram três ou mais reprovações, apresentaram um risco duas vezes maior de ser usuários de drogas, mesmo após o ajuste para fatores socioeconômicos e demográficos. Ainda assim, não seria possível estabelecer relação causal, pois tanto o uso de drogas poderia interferir no bom desempenho do aluno, como dificuldades escolares poderiam tornar-se fatores de risco para uso de drogas.

A associação da dependência química com escolas públicas contraria os achados das pesquisas de Guimarães, Godinho, Cruz, Kappann e Junior (2004) e Soldera e cols. (2004), que demonstraram, pela comparação entre as redes pública e privada, maior prevalência de uso das drogas nas escolas particulares, o que, para os investigadores, poderia estar ligado à condição social, cuja disponibilidade de recursos financeiros facilitaria a aquisição de drogas.

A maioria dos adolescentes com DQ mencionaram que muitos amigos fazem uso de drogas e os mesmos oferecerem-nas, o que corrobora a forte influência do grupo de pares nos adolescentes e a necessidade destes de sentir-se pertencentes a alguma "tribo". Rigoni, Oliveira e Andretta (2006) e Scivoletto e Morihisa (2001) afirmam que os fatores de risco que mais influenciam os adolescentes e favorecem à experimentação de substâncias psicoativas são: a curiosidade natural dos adolescentes, impulsionando-os a experimentar novas sensações e prazeres; a opinião de amigos, modismo, fácil acesso às drogas e oportunidade de uso, bem como o ambiente propício para a experimentação de drogas. Os achados desses pesquisadores estão de acordo com o presente estudo, indicando que a companhia e o incentivo dos amigos em relação à experimentação e ao uso de drogas pode ser um forte preditor para a dependência das mesmas.

Psico-USF, v. 12, n. 2, p. 269-279, jul./der. 2007 
Em relação à prática religiosa dos participantes deste estudo, divergindo de pesquisas atuais (Chaturvedi e cols., 2003; Sanchez e cols., 2004), não foi estatisticamente significativa à associação da religiosidade com o fato do adolescente não ser dependente químico. Deve-se pensar que o resultado deu-se em razão do número restrito de participantes, o que não torna os dados conclusivos.

Por fim, outro fator importante é a prevalência do uso do crack entre os participantes com dependência química. Sabe-se que esta é uma droga de custo relativamente baixo, se comparada às outras, e com alto poder de dependência, o que poderia explicar a alta incidência do uso da mesma entre os participantes com dependência química. A detecção do uso associado de maconha, cocaína e crack, fortemente presente nos participantes com dependência química, parece ser de grande importância, já que ainda são escassas as pesquisas que correlacionam adolescência e uso do crack. Ainda assim, sublinha-se que os dados extraídos desta pesquisa referem-se às particularidades e limitações do estudo em questão, sendo que não se pode, de forma alguma, fazer qualquer tipo de generalização a partir destes achados.

Marques e Cruz (2000) relatam que o consumo de drogas apresenta uma progressão em estágios, iniciando-se com o consumo de bebidas alcoólicas fermentadas e passando para o consumo de drogas ilícitas como a maconha e o crack nos estágios finais. $\mathrm{O}$ aumento da prevalência dos produtos derivados da coca, de acordo com Nappo, Galduróz e Carlini (2000), ocorre pela alta disponibilidade do rrack e seu baixo preço. Desse modo, parece ser de fundamental importância o desenvolvimento de estudos e de ações preventivas que compreendam de que forma a produção e o tráfico de drogas têm atuado, já que se sabe que as drogas têm sido desenvolvidas com o objetivo de tornar seus usuários dependentes de forma rápida, para que sejam garantidas a clientela e a movimentação desse mercado mundial milionário.

\section{Considerações finais}

Este estudo foi desenvolvido prioritariamente visando ao levantamento de dados em relação à dependência química na adolescência, bem como à comparação das percepções dos adolescentes com e sem diagnóstico de dependência química em relação às práticas educativas de seus pais.

Ao longo da pesquisa foram detectadas algumas limitações que não permitem que os achados sejam generalizados. O número pequeno de participantes, bem como o fato de os adolescentes sem diagnóstico de dependência química terem sido acessados pela rede social da pesquisadora, foram variáveis limitadoras do estudo. Outra limitação reside no fato de não terem sido incluídas as drogas lícitas na análise, uma vez que o álcool e o tabaco, duas fortes representantes dessa classe de drogas, são substâncias de fácil acesso e, como é sabido, amplamente utilizadas pelos jovens, ainda que ilícitas para os menores de 18 anos.

Sugestão para outro estudo com a mesma abordagem e delineamento semelhante, além dos cuidados com as limitações anteriormente citadas, seria analisar as diferenças entre a percepção das práticas parentais maternas e paternas, uma vez que a literatura ressalta que diferenças nas práticas educativas entre o casal parental também podem gerar comportamentos de risco.

\section{Referências}

Alvarenga, P. (2001) Práticas educativas parentais como forma de prevenção de problemas de comportamento. Em H. J. Guilhardi (Org.). Sobre o comportamento e cognição (vol. 8, pp. 54-60). Porto Alegre: ESETec.

Alvarenga, P. \& Piccinini, C. (2001). Práticas educativas e problemas de comportamento em pré-escolares. Psicologia: Reflexão e Crítica, 14(3), 449-460.

Associação Psiquiátrica Americana - APA (1995). Manual Diagnóstico e Estatístico de Transtornos Mentais - DSM-IV (D. Batista, Trad.). Porto Alegre: Artes Médicas.

Aunola, K, Stattin, H. \& Nurmi, J. E. (2000). Parenting styles and adolescents' achievement strategies. Journal of Adolescence, 23(2), 205-22.

Baumrind, D. (1966). Effects of authoritative parental control on child behavior. Child Development, 37(4), 887907.

Baus, J., Kupek, E. \& Pires, M. (2002). Prevalência e fatores de risco relacionados ao uso de drogas entre adolescentes. Revista de Saúde Pública, 36(1), 40-46.

Carlini, E., Galduróz J., Noto A. \& Nappo S. (2001). I levantamento domiciliar sobre o uso de drogas no Brasil. Centro Brasileiro de Informações sobre Drogas Psicotrópicas - Departamento de Psicobiologia da Escola Paulista de Medicina e SENAD - Secretaria Nacional Antidrogas, Presidência da República, Gabinete de Segurança Nacional.

Carr, M. B. \& Vandiver, Y. A. (2001) Risk and protective factors among youth offenders. Adolescence, 36(143), 409-426.

Chaturvedi, H. K., Phukan, R. K. \& Mahanta, J. (2003). The association of selected sociodemographic factors ans differences in patterns of substance use: A pilot study in selected areas of Northeast India. Substance Use \& Misuse, 38(9), 1305-1322.

Cohen, D. A. \& Rice, J. (1997). Parenting styles, adolescent substance use and academic achievement. Journal of Drug Education, 27(2), 199-211. 
Costa, F. T., Teixeira, M. A. P. \& Gomes, W. B. (2000). Responsividade e exigência: duas escalas para avaliar estilos parentais. Psicologia: Reflexão e Crítica, 13(3), 465473.

Darling, N. \& Steinberg, L. (1993). Parenting style as a context: An integrative model. Psychological Bulletin, 113(3), 487-496.

Dekovic, M., Janssens, J. A. M. \& Van As, N. M. C. (2003). Family predictors of antisocial behavior in adolescence. Family Process, 42(2), 223-235.

De Micheli, D. \& Formigoni, M. L. (2001). As razões para o primeiro uso de drogas e as circunstâncias familiares prevêem os padrões de uso futuro? Jornal Brasileiro de Dependência Química, 2(1), 20-30.

Duvicq, C. G., Pereira N. R. \& Carvalho A. M. (2004). Consumo de drogas lícitas e ilícitas en escolares y factores de protección y riesgo. Revista Latino-Americana de Enfermagem, 12, 345-51.

Fischer, J. \& Corcoran, K. (1994). Measures for clinical practice: A sourcebook. New York: The Free Press.

Gomes, A. \& Resende, V. (2004). O pai presente: o desvelar da paternidade em uma familia contemporânea. Psicologia: Teoria e Pesquisa, 20(2), 119-125.

Gomide, P. (2003). Estilos parentais e comportamento anti-social. Em A. Del Prette \& Z. A. P. Del Prette (Orgs.). Habilidades sociais, desenvolvimento e aprendizagem (pp. 21-60). Campinas: Alínea.

Guimarães, J. L., Godinho, P. H., Cruz, R., Kappann, J. I. \& Junior, L. A. T. (2004). Consumo de drogas psicoativas por adolescentes escolares de Assis, SP. Revista de Saúde Pública, 38(1), 130-132.

Huesca, R. S., Cruz, V. M., Encinas, R. O. \& Pantoja, G. I. (2002). Detección temprana de factores de riesgo para el consumo de substancias ilícitas. Salud Mental, 25(3), 1-11.

Kessler, F., Diemen, L., Seganfredo, A. C., Brandão, I., Saibro, P., Scheidt, B. \& Ramos, S. P. (2003). Psicodinâmica do adolescente envolvido em drogas. Revista de Psiquiatria do Rio Grande do Sul, 1, 33-41.

Maccoby, E. \& Martin, J. (1983). Socialization in the context of the family: Parent-child interaction. Em E. M. Hetherington (Org.). Handbook of child psychology, Socialization, personality, and social development (vol. 4, pp. 1101). New York: Wiley.

Marques, A. C. \& Cruz, M. S. (2000). O adolescente e o uso de drogas. Revista Brasileira de Psiquiatria, 22(2), 32-36.

Miller, J. M., DiIorio C. \& Dudley, W. (2002) Parenting style and adolescent's reaction to conflict: Is there a relationship? Journal of Adolescent Health, 31(6), 463-8.
Nappo, S. A., Galduróz, J. C. F. \& Carlini, E. A. (2000). O uso de cocaína: relatório informante chave (IC) de São Paulo - Brasil. Jornal Brasileiro de Psiquiatria, 49(5), 14966.

Oliveira, E. A., Marin, A. H. \& Pires, F. B. (2002). Estilos parentais autoritário e democrático-recíproco intergeracionais, conflito conjugal e comportamentos de externalização e internalização. Psicologia: Reflexão e Crítica, 15(1), $1-11$.

Patock-Peckham, J. A., Cheong, J., Balhorn, M. E. \& Nagoshi, C. T. (2001). A social learning perspective: A model of parenting styles, self-regulation, perceived drinking control, and alcohol use and problems. Alcoholism: clinical and experimental research, 25(9), 1284-92.

Pechansky, F., Szobot, C. \& Scivoletto, S. (2004). Uso de álcool entre adolescentes: conceitos, características epidemiológicas e fatores etiopatogênicos. Revista Brasileira de Psiquiatria, 26(1), 14-17.

Pentz, M. A. (2003). Evidence baed prevention: Characteristics, impact and future direction. Journal of Psychoactive Drugs, 35(1), 143-152.

Piko, B. (2000). Perceived social support from parents and peers: Wich is the sronger predictor of adolescent substance use? Substance Use \& Misuse, 35(4), 617-631.

Pons, J. (1998). El modelado familiar y el papel educativo de los padres en la etiología del consumo de alcohol en los adolescentes. Revista Española de Salud Publica, 72(3), 251-266.

Rebolledo, E., Ortega, N. \& Pillon, S. (2004). Factores de riesgo asociados al uso de drogas en estudiantes adolescentes. Revista Latino-Americana de Enfermagem, 12, 369-375.

Rigoni, M., Oliveira, M. \& Andretta, I. (2006). Conseqüências neuropsicológicas do uso da maconha em adolescentes e adultos jovens: uma revisão da literatura científica recente. Ciências \& Cognição [On-line], 3(8). Obtido em 10 de novembro de 2006 do World Wide Web: http:/ / www.cienciasecognicao.org.

Salvador, A. P. \& Weber, L. N. (2005). Práticas educativas parentais: um estudo comparativo da interação familiar de dois adolescentes distintos. Interação em Psicologia, 2, 341-353.

Sanchez, Z. V., Oliveira, L. G. \& Nappo, S. A. (2004). Fatores protetores de adolescentes contra o uso de drogas com ênfase na religiosidade. Ciência e Saúde Coletiva, 9, 43-55.

Scivoletto, S. \& Morihisa, R. S. (2001). Conceitos básicos em dependência de álcool e outras drogas na 
adolescência. Jornal Brasileiro de Dependência Química, 2(1), 30-33.

Sidman, M. (2001). Coerção e suas implicaçoes. Campinas: Livro Pleno.

Soldera, M., Dalgalarrondo, P., Filho, H. R. C. \& Silva, C. A. M. (2004). Uso de drogas psicotrópicas por estudantes: prevalência e fatores sociais associados. Revista de Saúde Pública, 38(2), 277-283.

Steinberg, L., Darling, N. \& Fletcher, A. C. (1995). Authoritative parenting and adolescent adjustment: An ecological journey. Em P. Moen, G. H. Elder Jr. \& K. Luscher (Orgs.). Examining lives in context: Perspectives on the ecology of human development (pp. 423-466). Washington, DC: APA.

Steinberg, L., Lamborn, S. D., Darling, N., Mounts, N. S. \& Dornbusch, S. M. (1994). Over-time changes in adjustment and competence among adolescents from authoritative, authoritarian, indulgent, and neglectful families. Child Development, 65, 754-770.

Tavares, B. F, Béria, J. U. \& Lima, M. S. (2001). Prevalência do uso de drogas e desempenho escolar entre adolescentes. Revista de Saúde Pública, 35(2), 150 158.

Wagner, A. \& Bem, L. A. (2006). Reflexão sobre a construção da parentalidade e o uso de estratégias educativas em famílias de baixo nível sócio-econômico. Psicologia em Estudo, 2(1), 63-71.

Wagner, A., Grzybowski, L. \& Silveira, L. (2003). Estrategias educativas en la familia: una perspectiva transgeneracional. Revista del Congresso Bienal de Psicología en Santiago de Cuba, 101, Santiago, Cuba.

Wagner, A., Carpenedo, C., Melo, L. \& Silveira, P. (2005a). Estratégias de comunicação familiar: a perspectiva dos filhos adolescentes. Psicologia: Reflexão e Crítica, 18(2), 277-282.

Wagner, A., Predebon, J., Mossmann, C. \& Verza, F. (2005b). Compartilhar tarefas? Papéis e funções de pai e mãe na família contemporânea. Psicologia: Teoria $e$ Pesquisa, 21(2), 181-186.

Weber, L. N. D., Brandenburg, O. J. \& Viezzer, A. P. (2003). A relação entre o estilo parental e o otimismo da criança. Psico-USF, 8(1), 71-79.

Weber, L. N. D., Viezzer, P. \& Brandenburg, O. (2004a). $\mathrm{O}$ uso de palmadas e surras como prática educativa. Estudos de Psicologia, 9(2), 227-238.

Weber, L. N. D., Prado, P. M., Viezzer, A. P. \& Brandenburg, O. J. (2004b). Identificação de estilos parentais: o ponto de vista dos pais e dos filhos. Psicologia: Reflexão e Crítica, 17(4), 323-332.

Wu, N. S, Lu, Y., Sterling, S. \& Weisner, C. (2004). Family environment factors and substance abuse severity in an HMO adolescent treatment population. Clinical Pediatrics, 43, 323-333.

Recebido em maio de 2007 Reformulado em setembro de 2007 Aprovado em outubro de 2007

Sobre as autoras:

Carla Zart Broecker é psicóloga, terapeuta de Casal e Família, especialista em Dependência Química e mestre em Psicologia pela PUCRS.

Graciela Inchausti de Jou é psicóloga, doutora em Psicologia do Desenvolvimento pela Universidade Federal do Rio Grande do Sul (UFRGS) e professora visitante vinculada ao GIDEP do PPG em Psicologia da UFRGS. 
